\title{
Las extraordinarias formas de vida de los dinoflagelados del Pacífico mexicano
}

\author{
Sonia Jeanetthe Delgado del Villar, Rosalba Alonso Rodríguez* y \\ Laura Rebeca Jimenez-Gutierrez
}

\begin{abstract}
Resumen
Conforme se descubren nuevas especies, la humanidad toma conciencia de la riqueza y diversidad de la vida en la naturaleza. Particularmente en los ecosistemas marinos, nos damos cuenta que desconocemos todos los beneficios ecológicos y económicos que brinda el fitoplancton. En este documento mostraremos las extraordinarias formas de vida de los dinoflagelados marinos, quienes conforman el segundo grupo más abundante después de las diatomeas dentro del fitoplancton marino, el cual contribuye a la producción de oxígeno y forma parte de la base de las cadenas tróficas de todos los sistemas acuáticos. Los dinoflagelados sobresalen por poseer una gran variedad de formas, tamaños y adaptaciones para sobrevivir en condiciones adversas. A partir de ellos, se obtienen diversos productos útiles para la sociedad como suplementos alimenticios y medicamentos. Sin embargo, algunas especies producen toxinas, también llamadas ficotoxinas, que provocan daños a los ecosistemas acuáticos y representan riesgos a la salud humana. Actualmente, se conoce sólo una pequeña parte de la diversidad de los dinoflagelados, su potencial en la producción de compuestos y su uso en beneficio de la sociedad. Aún estamos muy lejos de conocer todo lo que este maravilloso mundo microscópico tiene por ofrecer a la humanidad.
\end{abstract}

Palabras clave: biodiversidad, ficotoxinas, fitoplancton, microalgas peligrosas, productores primarios.

\section{The extraordinary life forms of the dinoflagellate from the MeXican Pacific}

\begin{abstract}
As new species are discovered, humanity becomes aware of the richness and diversity of nature. In marine ecosystems, particularly, we do not know entirely the ecological and economic benefits that phytoplankton provides. In this document, we will show the extraordinary life forms of marine dinoflagellates, who make up the second most abundant group after diatoms within marine phytoplankton, which contributes to the oxygen production and that are part of the basis of trophic chains of all aquatic systems. Dinoflagellates stand out for having a great variety of shapes, sizes and adaptations to survive in adverse conditions. From them, various useful products for society are obtained, such as food supplements and drugs. However, some species produce toxins, also called phycotoxins, which cause damage to aquatic ecosystems and represent risks to human health. Currently, we only know a small part of the diversity of dinoflagellates, its potential in the production of compounds and its use for the benefit of society. We are still a long way from knowing all that this wonderful microscopic world has to offer to humanity.
\end{abstract}

Keywords: biodiversity, phycotoxins, phytoplankton, harmful microalgae, primary producers.

Recepción: 01/12/2020. Aprobación: 12/03/2021.

http://doi.org/10.22201/cuaieed.16076079e.2021.22.4.3 
Estudió la Maestría en Ciencias en la UNAM y la Ingeniería en Biotecnología por la Universidad Politécnica de Sinaloa. Actualmente es estudiante del Doctorado en Ciencias en Recursos Acuáticos en la Universidad Autónoma de Sinaloa. Sus líneas de investigación e intereses son las biotoxinas marinas, cultivo de microalgas, determinación de ficotoxinas en moluscos y fitoplancton.

Rosalba Alonso Rodríguez

*Autor de correspondencia rosalba@ola.icmyl.unam.mx orcid.org/0000-0001-7716-3869 scopus: Alonso-Rodríguez, R.

Licenciada en Biología Pesquera, maestra en Ciencias del Mary doctora en Uso, Manejoy Preservación de los Recursos Naturales. Cuenta con más de 15 años de experiencia laboral, desarrollando líneas de investigación sobre microalgas productoras de toxinas, sus efectos sobre organismos y riesgos para la salud humana. Está adscrita al Instituto de Ciencias del Mar y Limnología de la Universidad Nacional Autónoma de México (UNAM), Unidad Academica Mazatlán, Sinaloa, México.

\section{Laura Rebeca Jimenez-Gutierrez}

ljimenez@uas.edu.mx

Estudió el Doctorado en Ciencias por el Centro de Investigación en Alimentación y Desarrollo; la Maestría en Ciencias en Acuacultura por la Universidad de Sonora y es bióloga con especialidad acuática por el Tecnológico Nacional de México. Actualmente es Catedrática CONACYT, de la Facultad de Ciencias del Mar, Universidad Autónoma de Sinaloa, y es SNI nivel 1. Su líneas de investigación e intereses son la biología reproductiva, con especialidad en crustáceos decápodos. 
"Las extraordinarias formas de vida de los dinoflagelados del Pacífico mexicano" Sonia Jeanetthe Delgado del Villar, Rosalba Alonso Rodríguez y Laura Rebeca Jimenez-Gutierrez

Vol. 22, Núm. 4, julio-agosto 2021 Revista Digital Universitaria

\section{El fitoplancton y los dinoflagelados}

El fitoplancton es el conjunto de microrganismos unicelulares de origen vegetal que flota en los mares, ríos, lagos y lagunas. Estos organismos son fotoautótrofos, pues poseen pigmentos fotosintéticos y realizan la fotosíntesis en los cloroplastos, producen glucosa y materia orgánica a partir del dióxido de carbono, materia inorgánica (nutrientes), agua y luz solar, y como producto de la reacción liberan oxígeno. Estos organismos constituyen la base de la cadena alimenticia, lo que conlleva al inicio de la transferencia de la energía de una especie a otra, hasta llegar a los depredadores de gran tamaño. Por lo tanto, el fitoplancton es indispensable para la vida en la Tierra.

El fitoplancton contribuye a la biodiversidad del planetay se clasifica en varios grupos según sus características. En orden de abundancia en los ecosistemas marinos se encuentran las diatomeas, dinoflagelados, silicoflagelados, cocolitofóridos, cianobacterias, entre otros grupos. Cada especie requiere condiciones ambientales específicas para su crecimiento, como temperatura, luz, nutrientes, salinidad, entre otras, lo que determina su distribución geográfica. En condiciones específicas, las poblaciones del fitoplancton pueden alcanzar tal abundancia que son capaces de cambiar el color del agua, a lo cual se le conoce como florecimiento algal, conocido también como marea roja.

Aproximadamente $70 \%$ de estos florecimientos algales son capaces de promover la productividad acuática marina (Cortés-Altamirano et al., 2019). El resto de los florecimientos algales puede provocar diversos problemas en el ecosistema, como el elevado consumo de oxígeno disuelto en ausencia de luz o cuando finaliza el florecimiento, sumado, en algunos casos, a la producción de ficotoxinas, espuma y sustancias viscosas. El 52\% de las especies formadoras de estos eventos conocidos como florecimientos algales nocivos (FAN) pertenecen a los dinoflagelados marinos (IOC-UNESCO, 2009), que son responsables de 46\% del número total de FAN registrados a nivel mundial desde 1770 a la fecha (HAEDAT, 2021).

Los dinoflagelados son un grupo de microalgas unicelulares. Su nombre proviene de la palabra griega dino que significa giro y del latín flagellum que significa flagelo o látigo. Este grupo existe desde hace 1600 millones de años y durante ese tiempo ha evolucionado y sobrevivido a las adversidades y a las grandes extinciones (Meng et al., 2005). Tiene la capacidad de producir toxinas, que en concentraciones altas puede provocar mortandad de organismos e intoxicaciones en los ecosistemas acuáticos y en humanos por el consumo de mariscos contaminados; por otra parte, estudios científicos han logrado descubrir aplicaciones médicas para dichas toxinas (Assunção, 2021).

El objetivo de este artículo es difundir la gran diversidad de formas, estructuras y adaptaciones de las especies de dinoflagelados que habitan en el océano Pacífico mexicano (OPM), con la finalidad de generar interés en su estudio. Esto podría abrir nuevos campos de conocimiento que nos permitan entender su biología, cultivo, formación de FAN, la producción y aplicación de toxinas en beneficio de la sociedad. 


\section{¿Quiénes son los dinoflagelados?}

La mayor parte de los dinoflagelados habitan en la superficie del océano. Algunas especies viven solitarias, mientras que otras forman colonias (figura 1, A, B). Un menor número de especies vive en simbiosis, con otros organismos como los corales, diatomeas o microzooplancton, ya sea junto a ellos o dentro de ellos (Gómez, 2012).
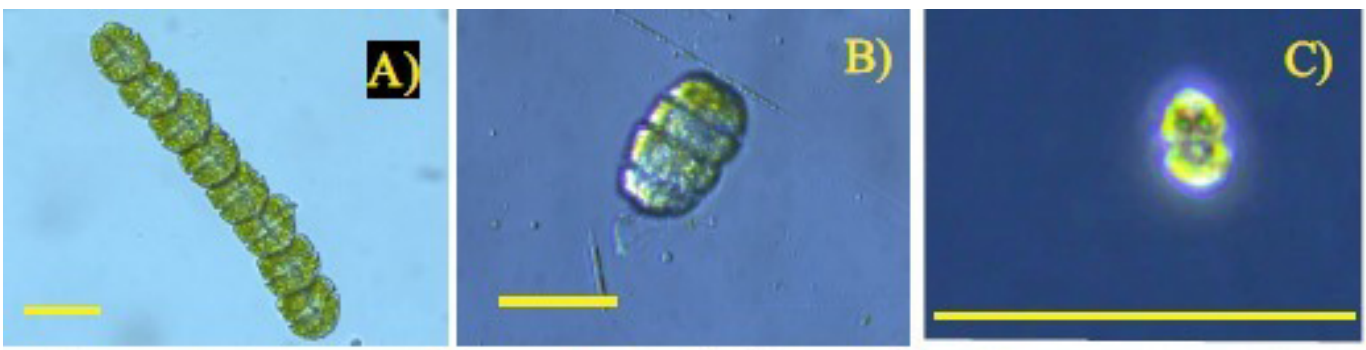

Figura 1. Diversidad de
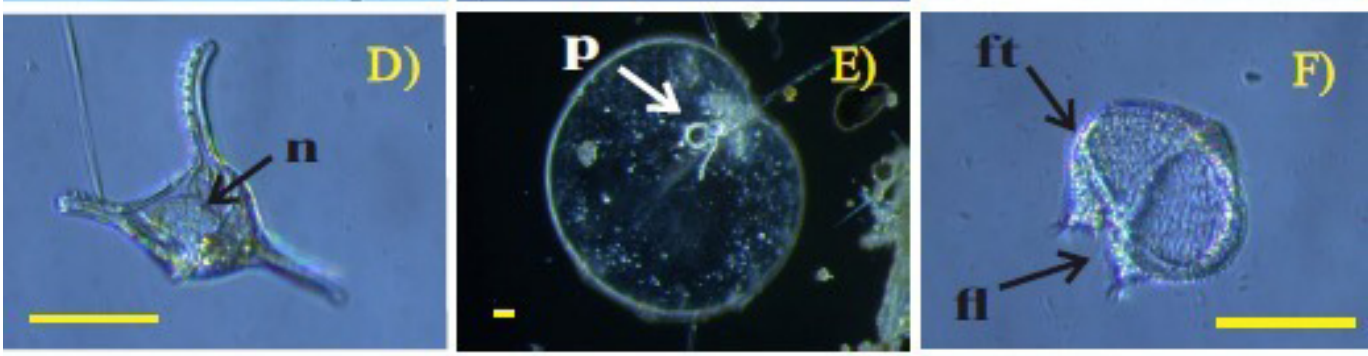

A) Cadena de Gymnodinium

catenatum. B) Pseudocolonia

de Polykrikos harmannii. C)

Heterocapsa sp. D) Ceratium tripos. E) Noctiluca scintillans. F)

Protoperidinium sp. G) Tripos

furca. H) Akashiwo sanguinea. I)

Quiste temporal de Phyrophacus

sp. J) Protoperidinium sp. K)

Noctiluca scintillans ingiriendo

cadenas de $G$. catenatum.

L) Prorocentrum concavum. $c a=$ cuerpo de acumulación cdi=cadena de dinoflagelado ingerida, ef= espina y flagelo,

$\mathrm{fl}=$ flagelo longitudinal, $\mathrm{ft}=$ flagelo transversal, n= núcleo, p=pedúnculo. La línea amarilla corresponde a la escala de 50 $\mu \mathrm{m}$. Elaboración propia.
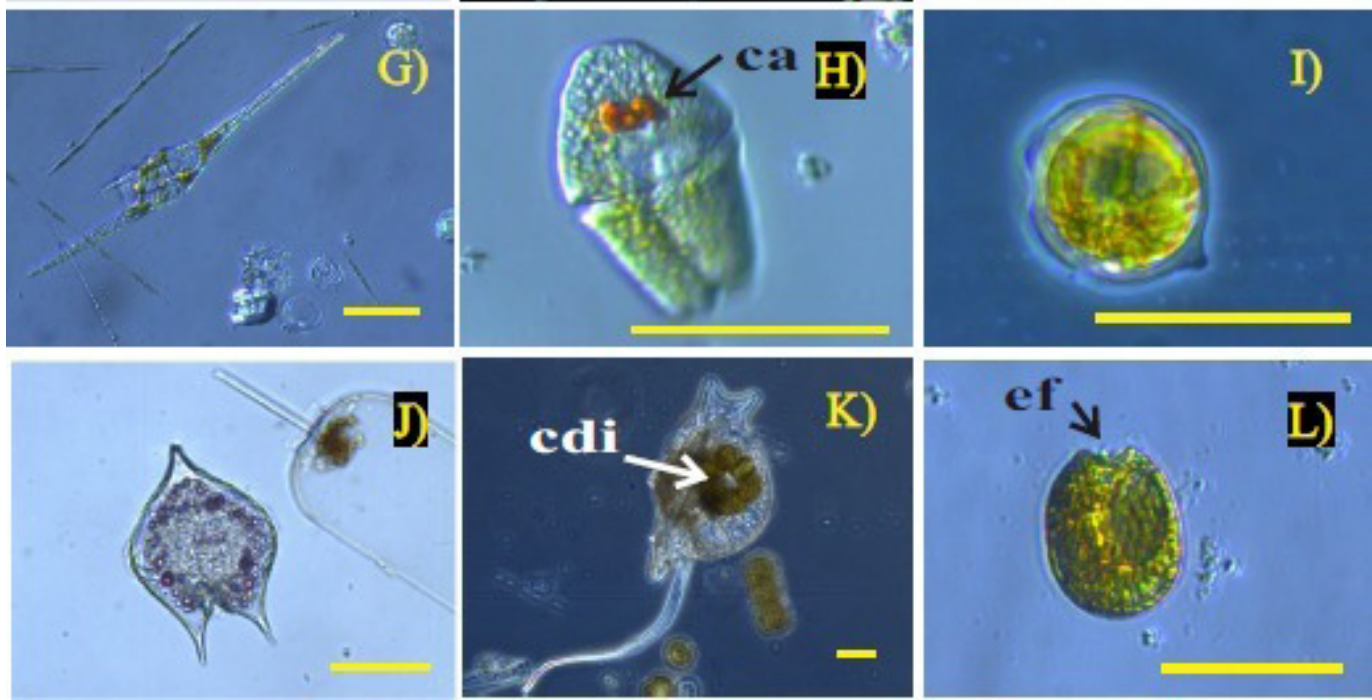

Los dinoflagelados tienen una gran diversidad de formas y tamaños, pueden

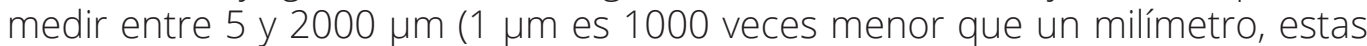
especies se observan a través de un microscopio; ver figura 1, C, D ,E). Algunas de estas variaciones corresponden a adaptaciones ambientales o a la etapa de su ciclo de vida (Gómez, 2012). Sin embargo, una de las características principales que comparten todos los dinoflagelados son sus dos flagelos (prolongación móvil) que facilitan su movimiento (ver figura 1F y figura 2; De Vargas et al., 2015). 
Figura 2. Características morfológicas de algunos géneros de dinoflagelados. A) Protoperidinium. B) Gymnodinium. C) Scrippsiella. D) Prorocentrum. $\mathrm{fl}=$ flagelo longitudinal, $\mathrm{ft}=$ flagelo transversal, n= núcleo, s= sulcus, st=sutura. Elaboración propia.
A)

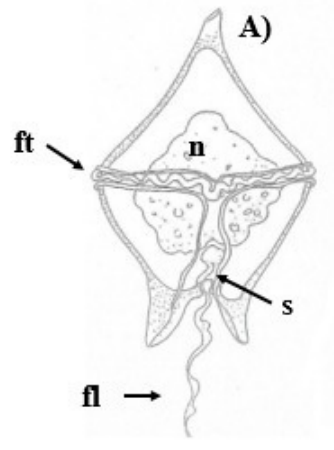

B)

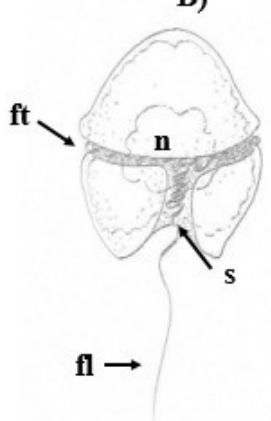

C)

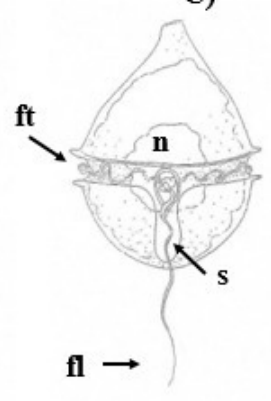

D)

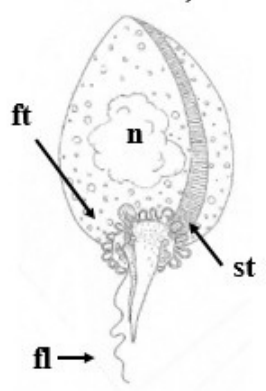

La otra razón del nombre de dinoflagelado es que su material genético permanece condensado en un núcleo grande denominado dinocarion. La mayoría de las especies de dinoflagelados poseen una pared celular delgada y son llamados atecados o desnudos, sólo en una cuarta parte de ellos esta pared presenta un aspecto rígido y se conocen como tecados. Algunas especies exhiben extensiones denominadas cuernos, también, pueden tener prolongaciones como son espinas, bordes, rebordes, alas, aletas o expansiones desiguales, etcétera (ver figura 1G; Hoppenrath et al., 2013).

Los dinoflagelados pertenecen a los primeros grupos de los eucariotas. Están más relacionados con los humanos que con las bacterias, aunque con estas últimas comparten algunas características, ya que se pueden reproducir tanto asexual como sexualmente, lo cual les brinda una ventaja evolutiva, debido a la combinación de genes, y, por ende, una mejor adaptación (Figueroa y Bravo, 2005).

Las condiciones ambientales cambiantes y poco favorables han permitido que cerca de 500 especies de dinoflagelados tengan la capacidad de sobrevivir en condiciones adversas, al transformarse de una célula móvil a una célula inmóvil en reposo denominada quiste, cuya forma puede corresponder o no a la especie que lo originó. Además, en su interior puede observarse un cuerpo de acumulación rojo que es un indicador de que tiene vida (ver figura 1, H, I).

Este quiste puede regresar a su forma móvil al restablecerse las condiciones ambientales y cumplir con un período de reposo programado por su reloj biológico (mecanismo molecular interno que le permite coordinar su funcionamiento, utilizando la información del medio ambiente). Se conoce sobre la existencia de quistes de dinoflagelados en estado de latencia que han subsistido enterrados en el sedimento durante aproximadamente 100 años (Cuellar-Martínez et al., 2017).

Cada especie de dinoflagelado puede mostrar un color característico durante un florecimiento algal, determinado por el tipo de pigmento que posea o la combinación de ellos, resaltando las clorofilas a y c2 (colores verdes), $\beta$-caroteno (colores naranja-rojizos) y peridinina (pardo-dorado), siendo este 
"Las extraordinarias formas de vida de los dinoflagelados del Pacífico mexicano" Sonia Jeanetthe Delgado del Villar, Rosalba Alonso Rodríguez y Laura Rebeca Jimenez-Gutierrez

Vol. 22, Núm. 4, julio-agosto 2021

Revista Digital Universitaria

último un pigmento exclusivo de los dinoflagelados (ver figura $1 \mathrm{~J}$; Zapata et al., 2012). Adicionalmente algunas especies de dinoflagelados tienen la capacidad para producir bioluminiscencia, lo cual dota a este grupo de gran notoriedad.

A pesar de que, por definición, todos los organismos fotosintéticos son capaces de producir su propio alimento, siempre existe una excepción a la regla. En este sentido, las especies de dinoflagelados practican una amplia variedad en formas de nutrición, la mitad de las especies realizan fotosíntesis, mientras que la otra mitad se alimenta de otros microorganismos. Sin embargo, la mayoría de las especies de dinoflagelados también practica la combinación de ambas formas de nutrición, lo que se conoce como mixotrofía (Stoecker et al., 2017).

La capacidad de alimentación externa en los dinoflagelados heterótrofos requiere de estructuras especializadas como el sulcus (surco longitudinal en la célula de los dinoflagelados que aloja el flagelo longitudinal) y ciertas prolongaciones o ranuras que asemejan bocas (ver figura 1, L, K). Los dinoflagelados quese alimentan a través de prolongaciones pueden ingerir presas 10 veces más grandes que ellos mismos, y algunas especies como Prorocentrum micans pueden tener varias bocas y engullir presas por diferentes ubicaciones al mismo tiempo. Lo anterior conlleva el uso de diferentes estrategias para la posible captación de las presas y al mismo tiempo el evitar a los depredadores (Jeong et al., 2005).

Como estrategia adicional para su sobrevivencia, los dinoflagelados poseen la capacidad de producir diversos compuestos químicos llamados ficotoxinas, que pueden transferirse a través de la cadena trófica hasta el humano por el consumo de mariscos contaminados, lo que provoca intoxicaciones y, en algunos casos, la muerte. Afortunadamente se han descubierto ciertas propiedades benéficas de las microalgas y sus aplicaciones, principalmente en países como Estados Unidos de América, Rusia, Irán, China y Japón (Tsianta, 2020).

Así, algunos de estos estudios con dinoflagelados demuestran que compuestos tóxicos como la saxitoxina y la tetrodotoxina se usan en tratamientos contra el dolor; el ácido ocadaico contra el Alzheimer, diabetes, sIDA y cáncer; mientras que la yesotoxina posee actividad antitumoral contra el cáncer de pulmón, colon y mama (Assunção et al., 2017). Otros estudios prueban que algunas especies no productoras de toxinas como Scrippsiella spp. crecen rápidamente y producen una gran cantidad de ácidos grasos que pueden ser usados como suplemento alimenticio para el ser humano y organismos acuáticos en cultivo (Xu et al. 2020).

Si bien los dinoflagelados pueden brindar grandes beneficios a la salud humana, sólo Crypthecodinium cohnii es cultivada industrialmente en la comunidad europea. Esta especie produce ácido decosahexaenoico (DHA), el cual se usa para el enriquecimiento de fórmulas lácteas infantiles (Assunção et al., 2017). Por otro lado, Canadá cuenta con una patente para la obtención de un antioxidante a partir de Crypthecodinium spp. En México no se han establecido cultivos de dinoflagelados a 
"Las extraordinarias formas de vida de los dinoflagelados del Pacífico mexicano" Sonia Jeanetthe Delgado del Villar, Rosalba Alonso Rodríguez y Laura Rebeca Jimenez-Gutierrez

Vol. 22, Núm. 4, julio-agosto 2021

Revista Digital Universitaria

nivel industrial, sólo se comercializan algunas cepas pertenecientes a la colección de dinoflagelados marinos (CODIMAR) con fines de investigación científica, ya que estos organismos son difíciles de cultivar y cosechar.

Se podría implementar el uso de diversos compuestos obtenidos a partir de cultivos de dinoflagelados en cualquier parte del mundo, ya que el fitoplancton marino se distribuye en todos los océanos, en ambos hemisferios norte y sur. Las regiones con mayor diversidad de especies son las regiones tropicales, el océano Índico, los mares del archipiélago indonesio-australiano y el océano Pacífico ecuatorial (De Vargas et al., 2015). La diversidad de dinoflagelados disminuye hacia latitudes altas, de la zona tropical a la zona templada, aumentando ligeramente al acercarse a los polos (Righetti et al., 2019).

Hoy en día, a nivel mundial, se conocen 3000 especies de dinoflagelados y se calcula que hay más de 500 especies por descubrir (Appeltans et al., 2012). En particular, en el océano Pacífico mexicano (opm), se han registrado en total 605 especies que comprenden 91 géneros de dinoflagelados. Los más representativos son Protoperidinium, Ceratium, Dinophysis, Gonyaulax, Oxytoxum, Gymnodinium, Prorocentrum, Alexandrium, Ornithocercus y Amphidinium (Okolodkov y GárateLizárraga, 2006), por lo que en el opm se encuentra $20 \%$ de la diversidad global estimada de dinoflagelados.

Estudios recientes han reportado la diversidad de especies de dinoflagelados para algunas regiones del opm de la siguiente manera:

- 168 especies pertenecientes a 41 géneros en el noroeste (GárateLizárraga et al., 2007).

- 211 especies pertenecientes a 31 géneros en el océano Pacífico central (Esqueda-Lara y Hernández-Becerril, 2010).

- 102 especies pertenecientes a 31 géneros en el océano Pacífico tropical mexicano (Torres et al., 2019).

Sin restar importancia al gran número de publicaciones sobre los dinoflagelados en México, es necesario ampliar los estudios sobre las formas de vida y diversidad de estos organismos, para sentar las bases de su aplicación.

\section{Conclusiones}

La información con que se cuenta acerca de las estrategias evolutivas de los dinoflagelados, como su morfología, reproducción, tipos de alimentación, capacidad de formar quistes, producción de toxinas y bioluminiscencia, nos habla de lo importante que es la preservación de los ecosistemas silvestres. Dado que algunas especies no producen toxinas, pueden ser usadas para alimentar a otros organismos acuáticos en cultivo o en la producción de suplementos alimenticios; mientras que las especies productoras de toxinas pueden ser usadas para la obtención de diversos compuestos para múltiples aplicaciones. Con un total de 
"Las extraordinarias formas de vida de los dinoflagelados del Pacífico mexicano"

Sonia Jeanetthe Delgado del Villar, Rosalba Alonso Rodríguez y

Laura Rebeca Jimenez-Gutierrez

Vol. 22, Núm. 4, julio-agosto 2021

Revista Digital Universitaria

1332 millones de kilómetros cúbicos de agua marina en el planeta y con más de 11,000 km de litoral mexicano, ¿cuántas especies más seremos capaces de descubrir?

\section{Referencias}

* Assunção, J., Guedes, A. C. y Malcata, F. X. (2017). Biotechnological and pharmacological applications of biotoxins and other bioactive molecules from dinoflagellates. Marine Drugs, 15, 393. http://doi.org./10.3390/md15120393

Appeltans, W., Ahyong, S. T., Anderson, G., Angel, A. V., Artois, T., Bailly, N., Bamber, R., Barber, A., Bartsch, I., Berta, A., Blazewicz-Paszkowycz, M., Bock, P., Boxshakk, G., Boyko, C. B., Brandäo, S. N., Bray, R. A., Bruce, N. L., Cairns, S. D., Chan, Chan, T. Y, ... Costello, M. J. (2012). The magnitude of global marine species diversity. Current Biology, 22(23), 2189-2202. https://doi.org/10.1016/j.cub.2012.09.036

* Cortés-Altamirano, R., Alonso-Rodríguez, R. y Salas-de-León, D. A. (2019). Historical observations of algal blooms in Mazatlan Bay, Sinaloa, Mexico (1979-2014). PLoS ONE, 14(1), e0210631. https://doi.org/10.1371/journal.pone.0210631

- Cuellar-Martínez, T., Ruiz-Fernández, A. C., Sánchez-Cabeza, J. A. y AlonsoRodríguez, R. (2017). Sedimentary record of recent climate impacts on an insular coastal lagoon in the Gulf of California. Quaternary Science Reviews, 160, 138-149. https://doi.org/10.1016/j.quascirev.2017.01.002

- De Vargas, C., Audic, S., Henry, N., Decelle, J., Mahe, F., Logares, R., Lara, E., Berney, C., Le Bescot, N., Probert, I., Carmichael, M., Poulain, J., Romac, S., Colin, S., Aury, J. M., Bittner, L., Chaffron, S., Dunthorn, M., Engelen, S., Flegontova, O., ... Karsenti, E. (2015). Eukaryotic plankton diversity in the sunlit ocean. Science, 348(6237), 1-12. http://dx.doi.org/10.1126/science.1261605

* Esqueda-Lara, K. y Hernández-Becerril, D. U. (2010). Dinoflagelados microplanctónicos marinos del Pacífico central de México (Isla Isabel, Nayarit y costas de Jalisco y Colima). Universidad Autónoma de México.

- Figueroa, R. I. y Bravo, I. (2005). Sexual reproduction and two different encystment strategies of Lingulodinium polyedrum (Dinophyceae) in culture. Journal of Phycology, 41, 370-379. https://doi.org/10.1111/j.1529-8817.2005.04150.x

* Gárate-Lizárraga, I., Band-Schmidt, C. J., Verdugo-Díaz, G., Muñeton-Gómez, M. S., Félix-Pico, E. F. (2007). Dinoflagelados (Dinophyceae) del sistema lagunar magdalena-almejas. En R. Funes Rodríguez, J. Gómez Gutiérrez y R. Palomares García (Eds.), Estudios Ecológicos en Bahía Magdalena (pp. 145-174). Gobierno del Estado de Baja California Sur; Fondo para la Protección de los Recursos Marinos de Baja California Sur; Instituto Politécnico Nacional; Centro Interdisciplinario de Ciencias Marinas. https://www.researchgate.net/publication/245536063_ Dinoflagelados_Dinophyceae_del_Sistema_Lagunar_Magdalena-Almejas

- Gómez, F. (2012). A quantitative review of the lifestyle, habitat and trophic diversity of dinoflagellates (Dinoflagellata, Alveolata). Systematics and Biodiversity, 10(3), 267275. http://dx.doi.org/10.1080/14772000.2012.721021 
"Las extraordinarias formas de vida de los dinoflagelados del Pacífico mexicano"

Sonia Jeanetthe Delgado del Villar, Rosalba Alonso Rodríguez y

Laura Rebeca Jimenez-Gutierrez

Vol. 22, Núm. 4, julio-agosto 2021

Revista Digital Universitaria

* Haedat. (2021). Harmful algae information system. http://haedat.iode.org/

* Hoppenrath, M., Chomérat, N., Horiguchi, T., Schweikert, M., Nagahama, Y. y Murray, S. (2013). Taxonomy and phylogeny of the benthic Prorocentrum species (Dinophyceae) - a proposal and review. Harmful Algae, 27, 1-28. https://doi. org/10.1016/j.hal.2013.03.006

* Jeong, H. J., Yoo, Y. D., Kim, J. S., Seong, K. A., Kang, N. S. y Kim, T. H. (2005). Growth, feeding and ecological roles of the mixotrophic and heterotrophic dinoflagellates in marine planktonic food webs. Ocean Science Journal, 45(2), 65-91. https://doi. org/10.1007/s12601-010-0007-2

* Meng, F.W., Zhou, C.M., Chen, Z.L. y Yuan, X.L. (2005). The oldest known dinoflagellates: Morphological and molecular evidence from Mesoproterozoic rocks at Yongji, Shanxi Province. Chinese Science Bulletin, 50(12), 1230-1234. http:// dx.doi.org/10.1007/BF03183698

* Moestrup, Ø., Akselmann-Cardella, R.; Churro, C.; Fraga, S.; Hoppenrath, M.; Iwataki, M.; Larsen, J.; Lundholm, N.; Zingone, A. (Eds.). (2009 onwards). IOC-UNEsco Taxonomic Reference List of Harmful Micro Algae. http://www.marinespecies.org/hab

* Okolodkov, Y. B. y Gárate-Lizárraga, I. (2006). An annotated checklist of dinoflagellates (Dinophyceae) from the Mexican Pacific. Acta Botanica Mexicana, 74(1), 1-154. http://dx.doi.org/10.21829/abm74.2006.1008

* Righetti, D., Vogt, M., Gruber, N., Psomas, A. y Zimmermann, N. E. (2019). Global pattern of phytoplankton diversity driven by temperature and environmental variability. Science Advances, 5(5), eaau6253. http://dx.doi.org/10.1126/sciadv. aau6253

- Tsianta, A. (2020). Pharmaceutical applications of Eukaryotic microalgae. [Tesis de maestría. International Hellenic University, Thessaloniki, Grecia]. https://repository. ihu.edu.gr/xmlui/bitstream/handle/11544/29668/a.tsianta.pdf?sequence=1

- Stoecker, D. K., Hansen, P. J., Caron, D. A. y Mitra, A. (2017). Mixotrophy in the marine plankton. Annual Review of Marine Science, 9, 311-335. http://dx.doi.org/10.1146/ annurev-marine-010816-060617

* Torres, G., Carnicer, O., Canepa, A., De La Fuente, P., Recalde, S., Narea, R., Pinto, E. y Borbot-Córdo, M. J. (2019). Spatio-temporal pattern of dinoflagellates along the Tropical Eastern Pacific Coast (Ecuador). Frontiers in Marine Science, 6, 145. https:// doi.org/10.3389/fmars.2019.00145

* Xu, S.J., Wu, K., Chan, S.C., Yau, Y., Chan, K. y Lee, F.W. (2020). Investigation of growth, lipid productivity, and fatty acid profiles in marine bloom-forming dinoflagellates as potential feedstock for biodiesel. Journal of Marine Science and Engineering, 8, 381. https://doi.org/10.3390/jmse8060381

* Zapata, M., Fraga, S., Rodríguez, F. y Garrido, J. L. (2012). Pigment-based chloroplast types in dinoflagellates. Marine Ecology Progress Series, 465, 33-52. http://dx.doi. org/10.3354/meps09879 


\section{Agradecimientos}

Sonia Jeanetthe Delgado del Villar agradece el apoyo otorgado por conACYT (beca \#335886). Los autores agradecen al equipo de trabajo del Laboratorio de Biotoxinas Marinas por el muestreo y registro fotográfico, al Lic. Rafael Serrano-Quiñonez y al Mat. Germán-Ramírez por la elaboración de figuras. Al Dr. Martín Frías Espericueta, por su revisión crítica del documento, y al Químico. Humberto Bojórquez Leyva por su apoyo en las salidas al campo. Este trabajo fue financiado por el Proyecto de Ciencia Básica A1-S-37026 "Caracterización de cepas de dinoflagelados potencialmente tóxicos aisladas del Pacífico mexicano en el contexto de Cambio Climático", el proyecto UNAM DGAPA PAPIIT IN112914 "Efecto de la resuspensión de sedimentos, nutrientes y temperatura en la formación de florecimientos algales nocivos por dinoflagelados productores de toxinas paralizantes en el Pacífico Mexicano", y el proyecto institucional Biotoxinas Marinas y sus efectos sobre organismos \#326 del ICMyL-UNAM, .

\section{Cómo CITAR ESTE ARTículo}

* Delgado-Villar, Sonia Jeanetthe, Alonso-Rodríguez Rosalba y Jimenez-Gutiérrez, Laura-Rebeca. (2021, julio-agosto). Las extraordinarias formas de vida de los dinoflagelados del Pacífico mexicano. Revista Digital Universitaria (rdu), 22(4). http:// doi.org/10.22201/cuaieed.16076079e.2021.22.4.3 